\title{
Performance of Spray Formulations on Morpho-Physiological Features and Growth of Orchid Cultivars
}

\author{
D. Das ${ }^{1}$, I. Kabir ${ }^{2}$ M. Islam ${ }^{3}$ \\ ${ }^{1}$ Assistant Professor, Agrotechnology Discipline, Khulna University, Khulna, \\ ${ }^{2}$ Senior Officer, Agrani Bank Ltd, Dhaka \\ ${ }^{3}$ Professor, Department of Crop Botany, Bangladesh Agricultural University, Mymensingh
}

\begin{abstract}
The experiment was conducted in the field laboratory of the Department of Crop Botany, Bangladesh Agricultural University (BAU), Mymensingh during the period of November, 2009 to September, 2010 to investigate the effect of spray formulations of NPK fertilizer on morphophysiological characters and growth of two orchid cultivars. The two factor experiment was laid out in the Completely Randomized Block Design (RCBD) with three replications. The two factors are cultivars (Mokara Diheard Red and Mokara Yellow Anne) and four spray formulations $\left(F_{0}, F_{1}, F_{2}, F_{3}\right)$. Data were collected on plant height, leaf length, leaf width, total leaf area, leaf area index, stem diameter, number of leaf, chlorophyll content, crop growth rate (CGR) and relative growth rate (RGR). Results revealed that the morphological and growth parameters of Mokara Diheard Red and Mokara Yellow Anne were significantly varied. Mokara Diheard Red performs better in chlorophyll content, CGR and RGR. Mokara Yellow Anne was superior in respect of leaf area index, total leaf area, leaf number and stem diameter. It was observed that the maximum increment of total leaf area, leaf area index, leaf number and stem diameter were found in spray formulation-3 $\left(F_{3}\right)$ and the lowest were in control $\left(F_{0}\right)$. Spray formulation-2 $\left(F_{2}\right)$ showed maximum increment of plant height, crop growth rate and relative growth rate over control $\left(F_{0}\right)$. So, the morpho-physiological features of orchid are significantly affected by spray formulation and the composition of spray formulation-2 $\left(F_{2}\right)$ and spray formulation-3 $\left(F_{3}\right)$ might be better for growth of orchid.
\end{abstract}

Key words: Orchid, Spray formulation, Mokara Diheard Red, Mokara Yellow Anne and growth

\section{Introduction}

Orchids are the most fascinating, varied and beautiful of all flowers due to its attractive structure and excellent color of petal (Singh and Voleti, 1995). Orchids having flowers of wonderful beauty and it has very good keeping qualities. They are the most wonderful items for indoor decoration (Patil, 2001). As cut flower and pot plants orchid is high demandable item. They are also used in different purposes as fragrant, glue, medicine, drinks and flavoring (Goh et al., 1992). There is a scope of large scale production of orchid in Bangladesh to meet the demand of international market and to earn foreign currency through export (Chowdhury, 1975). Orchid sticks produced in our country have great demand in local markets. The production can not meet the demand because of its slow growth. The growth of orchid is very slow and foliar nutrient application is very common practice in orchid cultivation as orchid cannot take nutrient significantly through root. Orchids possess aerial root which absorb moisture and nutrient from air. The growth of orchid can be enhanced by foliar feeding. Foliar spray nutrients mainly contain nitrogen $(\mathrm{N})$, phosphorus $(\mathrm{P})$, and potassium $(\mathrm{K})$ with different concentration. Application of spray nutrient containing N-P-K with different concentration varied on the basis of growth stage of plants. Urea, TSP and MoP are used as a source of nitrogen $(\mathrm{N})$, phosphorus $(\mathrm{P})$ and potassium $(\mathrm{K})$ in spray nutrients, respectively. Spray nutrients solution is prepared by mixing of Urea, TSP and MoP that plays important roles in growth and development of orchid. The schedule of application of spray formulation recommended by Bangladesh Agricultural Research Institute (BARI) is too much variable in every week that is very difficult to maintain. This problem can be overcome by rescheduling the time of application of spray formulation.

So, an experiment was undertaken to determine the effect of spray formulation on morphophysiological feature and growth of two orchid cultivars as well as to select suitable concentration of N, P and K in spray formulation for proper growth of orchid.

\section{Experimental Material}

\section{Materials And Method}

Two cultivars of orchid named Mokara Dihard Red and Mokara Yellow Anne were collected from Dipto orchid nursery, Valuka, Mymensingh. 


\section{Experimental Site}

The experiment was conducted in field laboratory of Crop Botany Department, Bangladesh Agriculture University, Mymensingh.

\section{Experimental Treatments} nutrients.

There are two factors used in this experiment known as variety and formulation of spray

Factor A: Two orchid cultivars viz. Mokara Diehard Red $\left(\mathrm{V}_{1}\right)$ and Mokara Yellow Anne $\left(\mathrm{V}_{2}\right)$

Factor B: Three levels of spray nutrient with control $\left(\mathrm{F}_{0}\right)$ viz, Spray Formulation $1\left(\mathrm{~F}_{1}\right)$, Spray Formulation $2\left(\mathrm{~F}_{2}\right)$, Spray Formulation $3\left(\mathrm{~F}_{3}\right)$

Thus, the total number of treatments was $8(4 \times 2)$ and each treatment was replicated three times. The spray solutions were prepared by mixing of Urea, Tripple Super Phosphate (TSP) and Muriate of Potash (MoP). Formulation was prepared by following the thumb rule of BARI where total amount of fertilizer to be $700 \mathrm{~g}$ per 172 litre water was recommended for use as spray. Spray formulation used in early stages contained higher N and gradually reduced in later stages.

Table1.Composition of spray formulation.

\begin{tabular}{|c|c|c|c|c|}
\hline Formulation & \multicolumn{3}{|c|}{ Total urea, TSP and MoP 70 g per 17.2 liter water at N : P : K ratio } \\
\hline $\mathrm{F}_{0}$ (Control) & 0.0 & 0.0 & 0.0 & 0.0 \\
\hline $\mathrm{F}_{1}$ & $15: 20: 25$ & $20: 20: 25$ & $20: 25: 30$ & $10: 30: 25$ \\
\hline $\mathrm{F}_{2}$ & $10: 15: 20$ & $15: 20: 25$ & $10: 25: 25$ & $10: 25: 30$ \\
\hline $\mathrm{F}_{3}$ & $5: 15: 10$ & $10: 15: 10$ & $10: 20: 15$ & $15: 20: 20$ \\
\hline $\begin{array}{c}\text { Duration of } \\
\text { spray }\end{array}$ & Up to 45 DAP & $\begin{array}{c}\text { From 45 DAP } \\
\text { to 90 DAP }\end{array}$ & $\begin{array}{c}\text { From 90 DAP } \\
\text { to 135 DAP }\end{array}$ & $\begin{array}{c}\text { From 135 DAP } \\
\text { to 180 DAP }\end{array}$ \\
\hline
\end{tabular}

$\%$ of N, P and $\mathrm{K}$ in Urea, TSP and MoP is 46.0, 48.0 and 60.0 respectively. In each times fresh formulation was prepared and sprayed once weekly with a hand sprayer in the afternoon.

\section{Collection of Data}

Data were collected periodically on Plant height $(\mathrm{cm})$, leaf length $(\mathrm{cm})$, leaf width $(\mathrm{cm})$, individual leaf area $\left(\mathrm{cm}^{2}\right)$, total leaf area $\left(\mathrm{cm}^{2}\right)$, leaf area Index (LAI), stem diameter $(\mathrm{cm})$, number of root/plant, number of leaf/ plant, chlorophyll content in leaf, fresh weight $(\mathrm{g})$ of plant sample and dry weight $(\mathrm{g})$ of plant sample.

\section{Experimental Design and Statistical Analysis}

The experiment was laid out in Completely Randomized Block Design (RCBD) with 8 treatments and each treatment was replicated three times. The collected data analyzed by following the analysis of variance (ANOVA) technique and mean differences were separated by Duncan's Multiple Range Test (DMRT) (Gomez and Gomez, 1984) using a computer operated programme named MSTAT-C (Russel, 1986).

\section{Morphological parameters Plant height}

\section{Results And Discussion}

Plant height significantly varied between the two cultivars of orchids. At 45 DAP, plant height of Mokara Diheard Red and Mokara Yellow Anne were $24.88 \mathrm{~cm}$ and $19.75 \mathrm{~cm}$, respectively. Maximum plant height of Mokara Diheard red and Mokara Yellow Anne were $32.19 \mathrm{~cm}$ and $27.44 \mathrm{~cm}$, respectively at 180 DAP (Table 1). It was observed that plant height increased progressively with the advancement of age and growth stages. Plant height increased rapidly in Mokara Yellow Anne than Mokara Diheard Red comparatively (Table1).The differences in plant height might be due to genetic makeup of orchid cultivars. Different nutrients of spray have significant affect on plant height. $F_{2}$ spray formulation performs better in plant height than other formulation (Table 2). Interaction effect of variety and spray formulation is significantly varied. Plant height increased rapidly in $\mathrm{V}_{2} \mathrm{~F}_{2}$ among all treatments (Table 3). So, it is said that composition of $\mathrm{F}_{2}$ spray formulation is mostly suitable for plant height. Kuksal et al. (1997) reported that nitrogen application at higher rates increased plant height. Plant height increased linearly with increase in $\mathrm{N}$, with application of $\mathrm{P}$ further increasing height (Hlgakl and Imamura, 1987). 


\section{Total Leaf Area}

Varietal effect of total leaf area was significant. The leaf area ranged from $523.55-1346.96 \mathrm{~cm}^{2}$ and $565.16-1540.38 \mathrm{~cm}^{2}$ in Mokara Diheard red and Mokara Yellow Anne, respectively over growing period (Table 1). From table it was observed that the total leaf area increment was higher in $V_{2}$ than $V_{1}$. Similarly, $F_{3}$ Spray formulation was much effective for enhancing the total leaf area (Table 2) and in case of interaction effect, maximum total leaf area increment was recorded in $\mathrm{V}_{2} \mathrm{~F}_{3}$ (Table 3). The variation in leaf area might occur due to the variation in number of leaves and their size which might be affected by spray formulation. The result obtained from the present study is consistent with the results of Sharma and Haloi (2001) who stated that variation in leaf area might be attributed to the difference in number of leaves.

\section{Leaf Area Index}

In comparison between two varieties degree of increasing of leaf area index was more in $V_{2}$ than $V_{1}$. Thus varietal effect of leaf area index was significant and $V_{2}$ may be considered as superior over $V_{1}$ in respect of leaf area index (Table 1). It was also observed that degree of increasing of leaf area index was more in $F_{3}$ spray formulation (Table 2) and in case of interaction effect, maximum leaf area index increment was recorded in $\mathrm{V}_{2} \mathrm{~F}_{3}$ (Table 3). The variation in leaf area index might occur due to the variation of leaf area and their expansion. As total leaf area was higher in $F_{3}$, so leaf area index also higher in $F_{3}$. The results obtained from the present study was consistent with the result of Sharma and Haloi (2001) who stated that variation in leaf area index could be attributed to the change in leaf number and leaf size. The results obtained from the present study also supported by Chandra and Das (2000).

\section{Leaf Number}

The leaf number ranged from 10.25-17.58 and 12.66-21.25 in Mokara Diheard red and Mokara Yellow Anne, respectively over growing period. Production of leaf number was higher in Mokara Yellow Anne than Mokara Diheard Red (Table 1). Similarly, Production of leaf number was higher in $\mathrm{F}_{3}$ spray formulation than other formulation (Table 2). Since level of nutrient concentrations in spray solution was altered over growth stage and number of leaves also increased with alteration of nutrient concentrations in spray formulation, so it might be concluded that spray nutrients have significant effect on leaf production. So, it is also said that composition of $\mathrm{F}_{3}$ spray formulation was very effective for growth of leaf. The present result supports the report of Singh and Kohli (1999). Leaf number varied significantly due to the interaction effect between variety and formulation. The variation in total number of leaves might be concerned with genetically variation, physiological function and growth characters of plant.

\section{Stem Diameter}

In Mokara Diheard Red, the average stem diameter per plant was 0.81 and $1.25 \mathrm{~cm}$ at 45 and 180 DAP, respectively. In Mokara Yellow Anne, the average stem diameter per plant was 0.96 and 1.43 at 45 and 180 DAP, respectively (Table 1). In comparison between two varieties degree of increasing of stem diameter was more in Mokara Yellow Anne than Mokara Diheard Red. $F_{3}$ spray formulation performs better for increasing stem diameter than other formulation which indicates that spray formulation containing $\mathrm{N}, \mathrm{P}$, and $\mathrm{K}$ have little effect on stem diameter (Table 2). The interaction between variety and formulation on stem diameter was varied significantly. Maximum increasing of stem diameter was recorded $\mathrm{V}_{2} \mathrm{~F}_{2}$ (Table 3).

\section{Physiological and Growth parameters \\ Chlorophyll Content}

At 90 DAP, the total chlorophyll content was higher in Mokara Diheard Red than Mokara Yellow Anne (Table 1). At 90 DAP, the total chlorophyll content $(22.73 \mathrm{mg} / \mathrm{g})$ was highest in $\mathrm{F}_{3}$ and the lowest was $11.96 \mathrm{mg} / \mathrm{g}$ in control $\left(\mathrm{F}_{0}\right)$, respectively (Table 2). The interaction between variety and spray formulation on total chlorophyll content was varied significantly. At 45 DAP, the highest total chlorophyll content $(25.3 \mathrm{mg} / \mathrm{g})$ was found in $\mathrm{V}_{1} \mathrm{~F}_{3}$ and the lowest $(8.68 \mathrm{mg} / \mathrm{g})$ was recorded in $\mathrm{V}_{1} \mathrm{~F}_{0}$. At $90 \mathrm{DAP}$, the highest total chlorophyll content $\left(26.31 \mathrm{mg} / \mathrm{g}\right.$ ) and the lowest chlorophyll content $\left(9.88 \mathrm{mg} / \mathrm{g}\right.$ ) were found in $\mathrm{V}_{1} \mathrm{~F}_{3}$ and $\mathrm{V}_{1} \mathrm{~F}_{0}$, respectively (Table 3).It is found that in all cases, chlorophyll content was lowest in control plants indicated that N, P and K in spray formulation has significantly affect on chlorophyll content.

\section{Crop Growth Rate}

The crop growth rate (CGR) in Mokara Diheard Red and Mokara Yellow Anne assessed from 45 to 180 DAP and it was observed that growth rate of orchid was very low. The crop growth rate of Mokara Diheard red was $0.29 \mathrm{~g} \mathrm{plant}^{-1} /$ day and of Mokara Yellow Anne was $0.17 \mathrm{~g} \mathrm{plant}^{-1} /$ day during the study period. Mokara Diheard red might be considered superior over Mokara Yellow Anne in respect of growth rate (Fig 1). Different spray formulations have significant effect on growth rate. The crop growth rate among different spray 
formulation ranged from $0.14-0.29 \mathrm{~g} \mathrm{plant}^{-1} /$ day. The maximum crop growth rate $\left(0.29 \mathrm{~g} \mathrm{plant}^{-1} /\right.$ day $)$ was found in $F_{2}$ spray formulation and the minimum crop growth rate $\left(0.14 \mathrm{~g} \mathrm{plant}^{-1} /\right.$ day) was in control $\left(\mathrm{F}_{0}\right)$ (Table 2) which indicated that spray nutrients formulation containing $\mathrm{N}, \mathrm{P}$ and $\mathrm{K}$ directly affected on crop growth rate. The interaction between variety and spray formulation on crop growth rate was varied significantly. The maximum crop growth rate $\left(0.37 \mathrm{~g}\right.$ plant ${ }^{-1} /$ day $)$ recorded from $\mathrm{V}_{1} \mathrm{~F}_{2}$ and the minimum crop growth rate $(0.12 \mathrm{~g}$ plant ${ }^{-1} /$ day) was recorded from $\mathrm{V}_{2} \mathrm{~F}_{0}$ (Table 3 ).

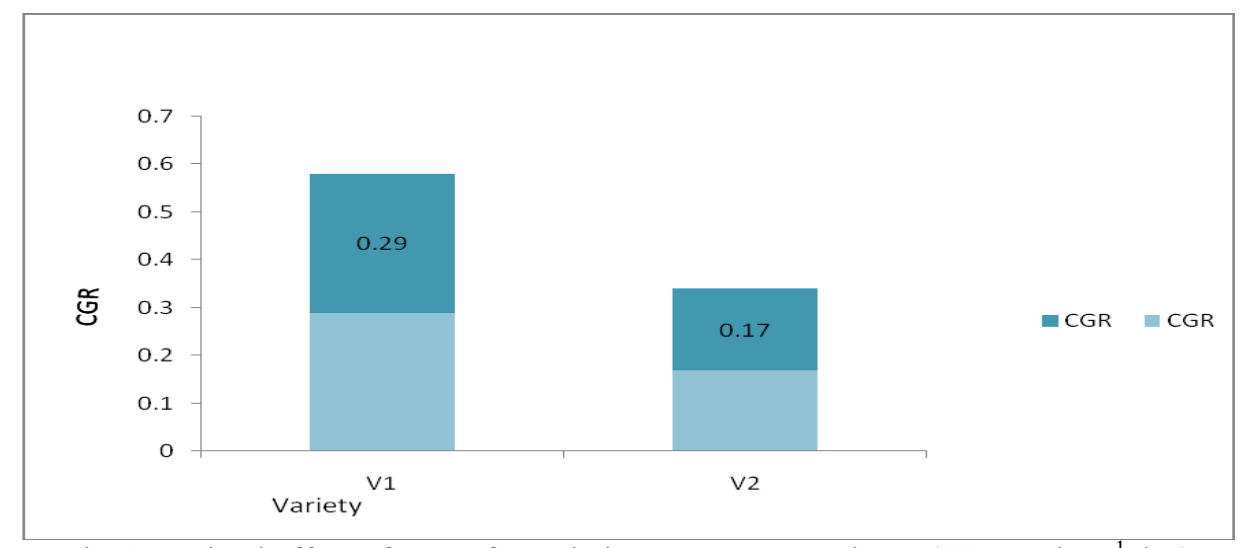

Fig.1: Varietal effect of spray formulation on crop growth rate(CGR gplant ${ }^{-1}$ day)

Mokara Diehard Red $=V_{1}$, Mokara Yellow Anne $=\mathrm{V}_{2}$

\section{Relative Growth Rate}

The relative growth rate of Mokara Diheard red was $6.88 \mathrm{mg} \mathrm{g}^{-1} /$ day and of Mokara Yellow Anne was $5.89 \mathrm{mg}$ $\mathrm{g}^{-1} /$ day (Table 1). Different spray formulations have significant effect on relative growth rate. The relative growth rate among different spray formulation ranged from $6.04-6.76 \mathrm{mg} \mathrm{g}^{-1} /$ day. The maximum relative growth rate was $\left(6.76 \mathrm{mg} \mathrm{g}^{-1} /\right.$ day) found in $F_{2}$ and the minimum $\left(6.04 \mathrm{mg} \mathrm{g}^{-1} /\right.$ day) was recorded from the control (Table 2 ) which indicated that spray formulation containing $\mathrm{N}, \mathrm{P}$ and $\mathrm{K}$ significantly affected the relative growth rate. The interaction between cultivars to spray formulation on relative growth rate was varied significantly. The maximum relative growth rate $\left(7.14 \mathrm{mg} \mathrm{g}^{-1} /\right.$ day $)$ was found in $\mathrm{V}_{2} \mathrm{~F}_{3}$ and minimum growth rate $\left(4.96 \mathrm{mg} \mathrm{g}^{-1} /\right.$ day $)$ was recorded from control $\mathrm{V}_{1} \mathrm{~F}_{0}$ (Table 3$)$

Table1. Main effect of variety on Plant height, total leaf area, leaf area index, leaf number, Stem diameter, CGR, RGR and total chlorophyll content Contd.

\begin{tabular}{|c|c|c|c|c|c|c|c|c|c|c|c|c|}
\hline \multirow[b]{2}{*}{$\begin{array}{l}\text { Variet } \\
\mathrm{y}\end{array}$} & \multicolumn{4}{|c|}{ Plant Height $(\mathrm{cm})$ at DAP } & \multicolumn{4}{|c|}{ Total leaf area $\left(\mathrm{cm}^{2}\right)$ at DAP } & \multicolumn{4}{|c|}{ Leaf number at DAP } \\
\hline & 45 & 90 & 135 & 180 & 45 & 90 & 135 & 180 & 45 & 90 & 135 & 180 \\
\hline $\mathrm{V}_{1}$ & 24.88 & 27.23 & 29.68 & 32.19 & 523.55 & 735.01 & 1029.82 & 1346.96 & 10.25 & 12.41 & 15.00 & 17.58 \\
\hline $\mathrm{V}_{2}$ & 19.75 & 22.28 & 24.95 & 27.44 & 565.16 & 809.6 & 1169.59 & 1540.38 & 12.66 & 15.33 & 18.41 & 21.25 \\
\hline $\mathrm{CV}$ & 16.91 & 14.63 & 12.88 & 12.09 & 11.62 & 14.41 & 12.91 & 12.08 & 14.69 & 11.77 & 10.37 & 8.48 \\
\hline LS & $* *$ & $*$ & $* *$ & $* *$ & $* *$ & $*$ & $*$ & ** & $* *$ & $* *$ & $* *$ & $* *$ \\
\hline
\end{tabular}

\begin{tabular}{|c|c|c|c|c|c|c|c|c|c|c|c|c|}
\hline \multirow[t]{2}{*}{ Variety } & \multicolumn{4}{|c|}{ Stem diameter $(\mathrm{cm})$ at $\mathrm{DAP}$} & \multirow{2}{*}{$\begin{array}{l}\text { CGR (g } \\
\text { plant } \\
1 / \text { day) }\end{array}$} & \multirow{2}{*}{$\begin{array}{l}\text { RGR } \\
\text { (mg g- } \\
1 / \text { day) }\end{array}$} & \multicolumn{2}{|c|}{$\begin{array}{l}\text { Total chlorophyll } \\
\text { content }(\mathrm{mg} / \mathrm{g})\end{array}$} & \multicolumn{4}{|c|}{ Leaf area index at DAP } \\
\hline & 45 & 90 & 135 & 180 & & & $\begin{array}{l}45 \\
\text { DAP }\end{array}$ & $\begin{array}{l}90 \\
\text { DAP }\end{array}$ & 45 & 90 & 135 & 180 \\
\hline $\mathrm{V}_{1}$ & 0.81 & 0.97 & 1.10 & 1.25 & 0.29 & 5.89 & 16.72 & 19.11 & 0.47 & 0.66 & 0.94 & 1.21 \\
\hline $\mathrm{V}_{2}$ & 0.96 & 1.11 & 1.27 & 1.43 & 0.17 & 6.88 & 13.32 & 17.9 & 0.5 & 0.74 & 1.05 & 1.39 \\
\hline $\mathrm{CV}$ & 13.56 & 11.23 & 8.37 & 8.12 & 17.82 & 8.50 & 0.44 & 2.03 & 11.62 & 14.25 & 12.61 & $\begin{array}{c}12.7 \\
7\end{array}$ \\
\hline LS & NS & NS & NS & NS & $* *$ & $* *$ & $* *$ & $* *$ & $* *$ & $* *$ & $*$ & $*$ \\
\hline
\end{tabular}


Table 2. Main effect of spray formulation on Plant height, total leaf area, leaf area index, leaf number, Stem diameter, CGR, RGR and total chlorophyll content Contd...

\begin{tabular}{|c|c|c|c|c|c|c|c|c|c|c|c|c|}
\hline \multirow{2}{*}{$\begin{array}{l}\text { Spray } \\
\text { formulation }\end{array}$} & \multicolumn{4}{|c|}{ Plant height $(\mathrm{cm})$ at DAP } & \multicolumn{4}{|c|}{ Total leaf area $\left(\mathrm{cm}^{2}\right)$ DAP } & \multicolumn{4}{|c|}{ Leaf area index at DAP } \\
\hline & 45 & 90 & 135 & 180 & 45 & 90 & 135 & 180 & 45 & 90 & 135 & 180 \\
\hline $\mathrm{F}_{0}$ & $\begin{array}{c}22.76 \\
b\end{array}$ & 24.20 & $\begin{array}{c}25.51 \\
b\end{array}$ & $\begin{array}{c}26.88 \\
b\end{array}$ & $\begin{array}{l}562.3 \\
\mathrm{a}\end{array}$ & $691 \mathrm{~b}$ & $\begin{array}{l}965.9 \\
b\end{array}$ & $\begin{array}{l}1298.0 \\
3 \mathrm{c}\end{array}$ & $\begin{array}{c}0.46 \\
\mathrm{a}\end{array}$ & $\begin{array}{c}0.63 \\
b\end{array}$ & $\begin{array}{c}0.89 \\
b\end{array}$ & $1.17 \mathrm{~b}$ \\
\hline $\mathrm{F}_{1}$ & $\begin{array}{c}24.15 \\
\mathrm{a}\end{array}$ & 25.91 & $28.8 \mathrm{a}$ & $\begin{array}{c}31.90 \\
\mathrm{a}\end{array}$ & $\begin{array}{l}549.0 \\
1 \mathrm{a}\end{array}$ & $\begin{array}{l}789.0 \\
6 \mathrm{ab}\end{array}$ & $\begin{array}{l}1135.1 \\
\mathrm{ab}\end{array}$ & $\begin{array}{l}1482.4 \\
2 \mathrm{a}\end{array}$ & $\begin{array}{c}0.49 \\
\mathrm{a}\end{array}$ & $0.71 \mathrm{a}$ & $\begin{array}{c}1.02 \\
\mathrm{a}\end{array}$ & $1.34 \mathrm{a}$ \\
\hline $\mathrm{F}_{2}$ & $\begin{array}{c}21.71 \\
b\end{array}$ & 24.45 & $\begin{array}{c}27.71 \\
a\end{array}$ & $\begin{array}{c}30.58 \\
\mathrm{a}\end{array}$ & $\begin{array}{l}558.0 \\
2 \mathrm{a}\end{array}$ & $\begin{array}{l}844.5 \\
9 \mathrm{a}\end{array}$ & $\begin{array}{l}1160.4 \\
6 \mathrm{a}\end{array}$ & $\begin{array}{l}1470.2 \\
\mathrm{a}\end{array}$ & $\begin{array}{c}0.5 \\
\mathrm{a}\end{array}$ & $0.76 \mathrm{a}$ & $1.05 \mathrm{a}$ & $1.33 \mathrm{a}$ \\
\hline $\mathrm{F}_{3}$ & $\begin{array}{c}21.63 \\
b\end{array}$ & 24.46 & $\begin{array}{c}27.25 \\
a\end{array}$ & $29.9 \mathrm{a}$ & $\begin{array}{l}508.0 \\
9 \mathrm{~b}\end{array}$ & $\begin{array}{l}764.5 \\
7 \mathrm{ab}\end{array}$ & $\begin{array}{l}1137.3 \\
7 \mathrm{ab}\end{array}$ & $\begin{array}{l}1524.0 \\
3 \mathrm{a}\end{array}$ & $\begin{array}{c}0.45 \\
\mathrm{a}\end{array}$ & $0.68 \mathrm{a}$ & $\begin{array}{c}1.02 \\
\mathrm{a}\end{array}$ & $1.37 \mathrm{a}$ \\
\hline $\mathrm{CV}$ & 16.91 & 14.63 & 12.88 & 12.09 & 11.62 & 14.41 & 12.91 & 12.08 & $\begin{array}{c}11.6 \\
2\end{array}$ & 14.25 & 12.61 & 12.77 \\
\hline LS & $*$ & NS & $*$ & $* *$ & $*$ & $* *$ & $*$ & $*$ & NS & $*$ & $*$ & $* *$ \\
\hline
\end{tabular}

Legend: $\mathrm{V}_{1}=$ Mokara Diheard Red, $\mathrm{V}_{2}=$ Mokara Yellow Anne, DAP = Days After Planting, Spray Formulation $1\left(\mathrm{~F}_{1}\right)$, Spray Formulation $2\left(\mathrm{~F}_{2}\right)$, Spray Formulation $3\left(\mathrm{~F}_{3}\right)$, LS= Level of significant, $* *=$ Significant at $1 \%$ level, $*=$ Significant at $5 \%$ level,

\begin{tabular}{|c|c|c|c|c|c|c|c|c|c|c|c|c|}
\hline \multirow{2}{*}{$\begin{array}{l}\text { Spray } \\
\text { formula } \\
\text { tion }\end{array}$} & \multicolumn{4}{|c|}{ Leaf number at DAP } & \multicolumn{4}{|c|}{ Stem diameter $(\mathrm{cm})$ at DAP } & \multicolumn{2}{|c|}{$\begin{array}{l}\text { Total Chlorophyll } \\
\text { content }(\mathrm{mg} / \mathrm{g})\end{array}$} & \multirow{2}{*}{$\begin{array}{c}\text { CGR(g } \\
\text { plant }^{-} \\
1 / \text { day })\end{array}$} & \multirow[t]{2}{*}{$\begin{array}{c}\text { RGR (mg } \\
\mathrm{g}^{-1} / \text { day) }\end{array}$} \\
\hline & 45 & 90 & 135 & 180 & 45 & 90 & 135 & 180 & 45DAP & 90DAP & & \\
\hline $\mathrm{F}_{0}$ & $\begin{array}{l}11.83 \\
\mathrm{a}\end{array}$ & 13.50 & $\begin{array}{l}16.50 \\
a\end{array}$ & 19.50 & 0.81 & 1.0 & 1.16 & 1.27 & $9.02 \mathrm{~d}$ & $11.96 \mathrm{~d}$ & $0.14 \mathrm{c}$ & $6.04 \mathrm{~d}$ \\
\hline $\mathrm{F}_{1}$ & $\begin{array}{l}12.83 \\
\mathrm{a}\end{array}$ & 14.16 & $\begin{array}{l}17.86 \\
\mathrm{a}\end{array}$ & 19.33 & 0.96 & 1.08 & 1.21 & 1.37 & $17.1 \mathrm{~b}$ & $21.05 \mathrm{~b}$ & $0.21 \mathrm{~b}$ & $6.12 \mathrm{c}$ \\
\hline $\mathrm{F}_{2}$ & $\begin{array}{l}11.66 \\
\mathrm{a}\end{array}$ & 14.50 & $\begin{array}{l}17.16 \\
a\end{array}$ & 19.83 & 0.83 & 0.97 & 1.13 & 1.3 & $14.75 \mathrm{c}$ & $17.80 \mathrm{c}$ & $0.29 \mathrm{a}$ & $6.76 \mathrm{a}$ \\
\hline $\mathrm{F}_{3}$ & $\begin{array}{l}10.50 \\
b\end{array}$ & 13.33 & $15.00 \mathrm{~b}$ & 19.00 & 0.93 & 1.11 & 1.25 & 1.41 & $19.22 \mathrm{a}$ & $22.73 \mathrm{a}$ & $0.28 \mathrm{a}$ & $6.62 \mathrm{~b}$ \\
\hline $\mathrm{CV}$ & 14.69 & 11.77 & 10.37 & 8.48 & 13.56 & 11.23 & 8.37 & 8.12 & 0.44 & 2.03 & 13.2 & 6.50 \\
\hline LS & $*$ & NS & $*$ & $\mathrm{NS}$ & NS & NS & NS & $\mathrm{NS}$ & $* *$ & $* *$ & $* *$ & $* *$ \\
\hline
\end{tabular}

Table 3.Interaction effect of variety to spray formulation on Plant height, total leaf area, leaf area index, leaf number, stem diameter, CGR, RGR and total chlorophyll content

\begin{tabular}{|c|c|c|c|c|c|c|c|c|c|c|c|c|}
\hline \multirow{2}{*}{$\begin{array}{l}\text { Variety } \mathrm{x} \\
\text { spray } \\
\text { formulati } \\
\text { on }\end{array}$} & \multicolumn{4}{|c|}{ Plant height $(\mathrm{cm})$ at DAP } & \multicolumn{4}{|c|}{ Total leaf area $\left(\mathrm{cm}^{2}\right)$ DAP } & \multicolumn{4}{|c|}{ Leaf area index at DAP } \\
\hline & 45 & 90 & 135 & 180 & 45 & 90 & 135 & 180 & 45 & 90 & 135 & 180 \\
\hline $\mathrm{V}_{1} \mathrm{~F}_{0}$ & $\begin{array}{c}25.46 \\
\mathrm{a}\end{array}$ & $\begin{array}{c}26.96 \\
\mathrm{a}\end{array}$ & $\begin{array}{c}28.10 \\
\mathrm{a}\end{array}$ & $\begin{array}{c}29.56 \\
b\end{array}$ & $613.7 \mathrm{ab}$ & $\begin{array}{c}756.19 \\
\text { bc }\end{array}$ & $\begin{array}{c}984.15 \\
\text { bc }\end{array}$ & $\begin{array}{c}1256.78 \\
b\end{array}$ & $\begin{array}{c}0.55 \\
\mathrm{ab}\end{array}$ & $\begin{array}{c}0.68 \\
\mathrm{~b}\end{array}$ & $\begin{array}{c}0.93 \\
\mathrm{bc}\end{array}$ & $\begin{array}{c}1.14 \\
\mathrm{~b}\end{array}$ \\
\hline $\mathrm{V}_{1} \mathrm{~F}_{1}$ & $\begin{array}{c}26.80 \\
\mathrm{a} \\
\end{array}$ & $\begin{array}{c}29.53 \\
\mathrm{a} \\
\end{array}$ & $\begin{array}{c}32.00 \\
\mathrm{a} \\
\end{array}$ & $\begin{array}{c}35.1 \\
\mathrm{a} \\
\end{array}$ & $478.0 \mathrm{c}$ & $\begin{array}{c}708.82 \\
\text { bc }\end{array}$ & $\begin{array}{c}1022.99 \\
\text { bc }\end{array}$ & $\begin{array}{c}1369.14 \\
a b\end{array}$ & $\begin{array}{c}0.43 \\
\mathrm{c}\end{array}$ & $\begin{array}{c}0.64 \\
\mathrm{~b} \\
\end{array}$ & $\begin{array}{c}0.92 \\
\mathrm{bc}\end{array}$ & $\begin{array}{c}1.24 \\
\mathrm{ab}\end{array}$ \\
\hline $\mathrm{V}_{1} \mathrm{~F}_{2}$ & $\begin{array}{c}24.40 \\
\mathrm{a}\end{array}$ & $\begin{array}{c}26.93 \\
\mathrm{a}\end{array}$ & $\begin{array}{c}30.0 \\
\mathrm{a}\end{array}$ & $\begin{array}{c}32.86 \\
\mathrm{a}\end{array}$ & $488.54 \mathrm{c}$ & $\begin{array}{c}688.64 \\
\text { bc }\end{array}$ & $\begin{array}{c}979.26 \\
\text { bc }\end{array}$ & $\begin{array}{c}1274.78 \\
\text { b }\end{array}$ & $\begin{array}{c}0.44 \\
\mathrm{c}\end{array}$ & $\begin{array}{c}0.62 \\
\mathrm{~b}\end{array}$ & $\begin{array}{c}0.88 \\
\mathrm{bc}\end{array}$ & $\begin{array}{c}1.15 \\
\mathrm{~b}\end{array}$ \\
\hline $\mathrm{V}_{1} \mathrm{~F}_{3}$ & $\begin{array}{c}22.86 \\
a b\end{array}$ & $\begin{array}{c}25.50 \\
\mathrm{a} \\
\end{array}$ & $\begin{array}{c}28.63 \\
\mathrm{ab}\end{array}$ & $\begin{array}{c}31.23 \\
\mathrm{a} \\
\end{array}$ & $\begin{array}{c}513.96 \\
\text { abc } \\
\end{array}$ & $\begin{array}{c}786.39 \\
\text { bc }\end{array}$ & $\begin{array}{c}1132.9 \\
\text { abc } \\
\end{array}$ & $\begin{array}{c}1487.13 \\
a b\end{array}$ & $\begin{array}{c}0.46 \\
\mathrm{bc}\end{array}$ & $\begin{array}{c}0.69 \\
\mathrm{~b}\end{array}$ & $\begin{array}{c}1.02 \\
\mathrm{bc}\end{array}$ & $\begin{array}{c}1.33 \\
\mathrm{ab}\end{array}$ \\
\hline $\mathrm{V}_{2} \mathrm{~F}_{0}$ & $\begin{array}{c}20.06 \\
b\end{array}$ & $\begin{array}{c}21.43 \\
b\end{array}$ & $\begin{array}{c}22.93 \\
b\end{array}$ & $\begin{array}{c}24.20 \\
b\end{array}$ & $\begin{array}{c}510.9 \\
\text { abc }\end{array}$ & $\begin{array}{c}625.80 \\
\mathrm{c}\end{array}$ & $947.66 \mathrm{c}$ & $\begin{array}{c}1339.29 \\
a b\end{array}$ & $\begin{array}{c}0.45 \\
\mathrm{bc}\end{array}$ & $\begin{array}{c}0.59 \\
b\end{array}$ & $\begin{array}{c}0.85 \\
\mathrm{c}\end{array}$ & $\begin{array}{c}1.21 \\
\mathrm{ab}\end{array}$ \\
\hline $\mathrm{V}_{2} \mathrm{~F}_{1}$ & $\begin{array}{c}19.50 \\
\mathrm{ab}\end{array}$ & $22.3 \mathrm{~b}$ & $\begin{array}{c}25.6 \\
a b\end{array}$ & $\begin{array}{c}28.70 \\
\mathrm{~b}\end{array}$ & $\begin{array}{c}620.02 \\
a b\end{array}$ & $\begin{array}{c}869.31 \\
\mathrm{ab}\end{array}$ & $\begin{array}{c}1247.20 \\
a b\end{array}$ & $\begin{array}{c}1595.7 \\
a b\end{array}$ & $\begin{array}{c}0.56 \\
\mathrm{a} \\
\end{array}$ & $\begin{array}{c}0.78 \\
\mathrm{ab}\end{array}$ & $\begin{array}{c}1.13 \\
\mathrm{ab}\end{array}$ & $\begin{array}{c}1.44 \\
\mathrm{ab}\end{array}$ \\
\hline $\mathrm{V}_{2} \mathrm{~F}_{2}$ & $\begin{array}{c}19.03 \\
\mathrm{~b}\end{array}$ & $\begin{array}{c}21.96 \\
\mathrm{~b}\end{array}$ & $\begin{array}{c}25.43 \\
\mathrm{ab}\end{array}$ & $\begin{array}{c}28.30 \\
\mathrm{~b}\end{array}$ & $627.50 \mathrm{a}$ & $\begin{array}{c}1000.5 \\
4 \mathrm{a} \\
\end{array}$ & $\begin{array}{c}1341.67 \\
\mathrm{a} \\
\end{array}$ & $\begin{array}{c}1665.61 \\
\mathrm{a} \\
\end{array}$ & $\begin{array}{c}0.56 \\
\mathrm{a} \\
\end{array}$ & $\begin{array}{c}0.90 \\
\mathrm{a} \\
\end{array}$ & $\begin{array}{c}1.21 \\
\mathrm{a} \\
\end{array}$ & $\begin{array}{c}1.51 \\
\mathrm{a} \\
\end{array}$ \\
\hline $\mathrm{V}_{2} \mathrm{~F}_{3}$ & $\begin{array}{c}20.40 \\
\mathrm{~b}\end{array}$ & $\begin{array}{c}23.43 \\
a b\end{array}$ & $\begin{array}{c}25.86 \\
a b\end{array}$ & $\begin{array}{c}28.56 \\
\mathrm{~b}\end{array}$ & $\begin{array}{c}502.22 \\
\text { bc }\end{array}$ & $\begin{array}{c}742.75 \\
\text { bc }\end{array}$ & $\begin{array}{c}1141.85 \\
a b c\end{array}$ & $\begin{array}{c}1560.93 \\
a b\end{array}$ & $\begin{array}{c}0.45 \\
\mathrm{bc}\end{array}$ & $\begin{array}{c}0.67 \\
\mathrm{~b}\end{array}$ & $\begin{array}{c}1.03 \\
b c\end{array}$ & $\begin{array}{c}1.41 \\
\mathrm{ab}\end{array}$ \\
\hline $\mathrm{CV}$ & 16.91 & 14.63 & 12.88 & 12.09 & 11.62 & 14.41 & 12.91 & 12.08 & 11.62 & 14.25 & $\begin{array}{c}12.6 \\
1 \\
\end{array}$ & 12.77 \\
\hline LS & $* *$ & * & $*$ & $*$ & $* *$ & $* *$ & $*$ & * & $* *$ & $*$ & $*$ & $* *$ \\
\hline
\end{tabular}

Legend: $\mathrm{V}_{1}=$ Mokara Diheard Red, $\mathrm{V}_{2}=$ Mokara Yellow Anne, DAP = Days After Planting, Spray Formulation $1\left(\mathrm{~F}_{1}\right)$, Spray Formulation $2\left(\mathrm{~F}_{2}\right)$, Spray Formulation $3\left(\mathrm{~F}_{3}\right), \quad \mathrm{LS}=$ Level of significant, $* *=$ Significant at $1 \%$ level, $*=$ Significant at $5 \%$ level, Contd. 


\begin{tabular}{|c|c|c|c|c|c|c|c|c|c|c|c|c|}
\hline \multirow{2}{*}{$\begin{array}{l}\text { Variety } x \\
\text { spray } \\
\text { formulatio } \\
n\end{array}$} & \multicolumn{4}{|c|}{ Leaf number at DAP } & \multicolumn{4}{|c|}{ Stem diameter $(\mathrm{cm})$ at DAP } & \multicolumn{2}{|c|}{$\begin{array}{c}\text { Chloro. } \\
\text { content }(\mathrm{mg} / \mathrm{g})\end{array}$} & \multirow{2}{*}{$\begin{array}{l}\text { CGR } \\
\text { (g } \\
\text { plant } \\
1 / \text { day })\end{array}$} & \multirow{2}{*}{$\begin{array}{c}\text { RGR } \\
(\mathrm{mg} \\
\mathrm{g}^{-} \\
1 / \text { day } \\
\text { ) }\end{array}$} \\
\hline & 45 & 90 & 135 & 180 & 45 & 90 & 135 & 180 & $\begin{array}{c}45 \mathrm{D} \\
\mathrm{AP}\end{array}$ & $\begin{array}{l}90 \mathrm{D} \\
\mathrm{AP}\end{array}$ & & \\
\hline $\mathrm{V}_{1} \mathrm{~F}_{0}$ & $12.0 \mathrm{abc}$ & $\begin{array}{l}13.33 \\
\text { bc }\end{array}$ & $\begin{array}{l}15.66 \\
b\end{array}$ & $\begin{array}{l}18.0 \\
\mathrm{~cd}\end{array}$ & $\begin{array}{l}0.75 \\
b\end{array}$ & $\begin{array}{l}0.93 \\
b\end{array}$ & $\begin{array}{l}1.11 \\
b\end{array}$ & $1.2 \mathrm{~b}$ & $\begin{array}{l}8.68 \\
h\end{array}$ & $\begin{array}{l}9.88 \\
\mathrm{~g}\end{array}$ & $\begin{array}{c}0.17 \\
\text { de }\end{array}$ & $\begin{array}{c}4.96 \\
\mathrm{~g}\end{array}$ \\
\hline $\mathrm{V}_{1} \mathrm{~F}_{1}$ & $9.0 \mathrm{c}$ & $\begin{array}{l}11.33 \\
\mathrm{c}\end{array}$ & $\begin{array}{l}14.33 \\
b\end{array}$ & $\begin{array}{l}16.66 \\
d\end{array}$ & $\begin{array}{l}0.88 \\
\mathrm{ab}\end{array}$ & $\begin{array}{l}1.0 \\
\mathrm{ab}\end{array}$ & $\begin{array}{l}1.08 \\
b\end{array}$ & $\begin{array}{l}1.26 \\
b\end{array}$ & $\begin{array}{l}17.7 \\
b\end{array}$ & $\begin{array}{l}20.52 \\
\mathrm{c}\end{array}$ & $\begin{array}{c}0.28 \\
\mathrm{bc}\end{array}$ & $\begin{array}{c}6.03 \\
\mathrm{f}\end{array}$ \\
\hline $\mathrm{V}_{1} \mathrm{~F}_{2}$ & $10.33 \mathrm{bc}$ & $\begin{array}{l}12.66 \\
\mathrm{c}\end{array}$ & $\begin{array}{l}15.33 \\
\mathrm{~b}\end{array}$ & $\begin{array}{l}17.66 \\
\mathrm{~cd}\end{array}$ & $\begin{array}{l}0.76 \\
\mathrm{~b}\end{array}$ & $\begin{array}{l}0.91 \\
\mathrm{~b}\end{array}$ & $\begin{array}{l}1.08 \\
\mathrm{~b}\end{array}$ & $1.2 \mathrm{~b}$ & $\begin{array}{l}15.21 \\
\mathrm{~d}\end{array}$ & $\begin{array}{l}19.79 \\
d\end{array}$ & $\begin{array}{c}0.37 \\
\mathrm{a}\end{array}$ & $\begin{array}{c}6.48 \\
\mathrm{c}\end{array}$ \\
\hline $\mathrm{V}_{1} \mathrm{~F}_{3}$ & $9.66 \mathrm{c}$ & $\begin{array}{l}12.33 \\
\mathrm{c}\end{array}$ & $\begin{array}{l}14.66 \\
b\end{array}$ & $\begin{array}{l}18.0 \\
\text { bcd }\end{array}$ & $\begin{array}{l}0.85 \\
\mathrm{ab}\end{array}$ & $\begin{array}{l}1.03 \\
\mathrm{ab}\end{array}$ & $\begin{array}{l}1.15 \\
b\end{array}$ & $\begin{array}{l}1.33 \\
\mathrm{ab}\end{array}$ & $\begin{array}{l}25.3 \\
\mathrm{a}\end{array}$ & $\begin{array}{l}26.31 \\
\mathrm{a}\end{array}$ & $\begin{array}{c}0.35 \\
\mathrm{ab}\end{array}$ & $6.1 \mathrm{e}$ \\
\hline $\mathrm{V}_{2} \mathrm{~F}_{0}$ & $\begin{array}{l}11.66 \\
\text { abc }\end{array}$ & $\begin{array}{l}13.66 \\
b c\end{array}$ & $\begin{array}{l}17.33 \\
\mathrm{ab}\end{array}$ & $\begin{array}{l}21.0 \\
a b\end{array}$ & $\begin{array}{l}0.88 \\
\mathrm{ab}\end{array}$ & $\begin{array}{l}1.06 \\
a b\end{array}$ & $\begin{array}{l}1.21 \\
\mathrm{ab}\end{array}$ & $\begin{array}{l}1.35 \\
a b\end{array}$ & $\begin{array}{l}9.36 \\
g\end{array}$ & $\begin{array}{l}14.05 \\
\mathrm{f}\end{array}$ & $\begin{array}{c}0.12 \\
\mathrm{e}\end{array}$ & $\begin{array}{c}7.13 \\
\mathrm{a}\end{array}$ \\
\hline $\mathrm{V}_{2} \mathrm{~F}_{1}$ & $14.66 \mathrm{a}$ & $17.0 \mathrm{a}$ & $20.0 \mathrm{a}$ & $22.0 \mathrm{a}$ & $\begin{array}{l}1.05 \\
\mathrm{a}\end{array}$ & $\begin{array}{l}1.16 \\
\mathrm{a}\end{array}$ & $\begin{array}{l}1.35 \\
\mathrm{a}\end{array}$ & $\begin{array}{l}1.48 \\
\mathrm{a}\end{array}$ & $\begin{array}{l}16.51 \\
c\end{array}$ & $\begin{array}{l}21.58 \\
b\end{array}$ & $\begin{array}{c}0.15 \\
\text { de }\end{array}$ & $\begin{array}{c}6.21 \\
d\end{array}$ \\
\hline $\mathrm{V}_{2} \mathrm{~F}_{2}$ & $13.0 \mathrm{ab}$ & $\begin{array}{l}16.33 \\
a b\end{array}$ & $19.0 \mathrm{a}$ & $22.0 \mathrm{a}$ & $\begin{array}{l}0.90 \\
a b\end{array}$ & $\begin{array}{l}1.03 \\
a b\end{array}$ & $\begin{array}{l}1.17 \\
a b\end{array}$ & $\begin{array}{l}1.4 \\
a b\end{array}$ & $\begin{array}{l}14.30 \\
e^{140}\end{array}$ & $\begin{array}{l}15.82 \\
e^{152}\end{array}$ & $\begin{array}{c}0.22 \\
\mathrm{~cd}\end{array}$ & $\begin{array}{c}7.05 \\
b\end{array}$ \\
\hline $\mathrm{V}_{2} \mathrm{~F}_{3}$ & $\begin{array}{l}11.33 \\
b c\end{array}$ & $\begin{array}{l}14.33 \\
a b c\end{array}$ & $\begin{array}{l}17.33 \\
\mathrm{ab}\end{array}$ & $\begin{array}{l}20.0 \\
a b c\end{array}$ & $\begin{array}{l}1.01 \\
\mathrm{a}\end{array}$ & $1.2 \mathrm{a}$ & $\begin{array}{l}1.35 \\
\mathrm{a}\end{array}$ & $1.5 \mathrm{a}$ & $\begin{array}{l}13.13 \\
\mathrm{f}\end{array}$ & $\begin{array}{l}19.15 \\
d\end{array}$ & $\begin{array}{c}0.21 \\
\mathrm{~cd}\end{array}$ & $\begin{array}{c}7.14 \\
\mathrm{a}\end{array}$ \\
\hline $\mathrm{CV}$ & 14.69 & 11.77 & 10.37 & 8.48 & 13.56 & 11.23 & 8.37 & 8.12 & 0.44 & 2.03 & 17.82 & 5.50 \\
\hline LS & $*$ & $* *$ & $*$ & $*$ & $*$ & $* *$ & *** & $* *$ & $* *$ & $* *$ & NS & $* *$ \\
\hline
\end{tabular}

\section{Summary And Conclusion}

The experiment was conducted in grill house of the Department of Crop Botany, BAU, during the period of 30 November, 2009 to 30 September, 2010 to determine the effect of spray nutrients on morpho-physiological features and growth of orchid. The experiment comprised of two orchid cultivars and four levels of spray nutrients with control. The experiment was laid out in Completely Randomized Block Design (RCBD) with 8 treatments and each treatment was replicated three times. Data were taken on some selected parameters and statistical analyses were performed by using MSTAT-C program. The results of the experiment were summarized below.

Results revealed that all the studied parameters were significantly influenced by spray formulation consists of N, P and K. Results showed that morpho-physiological features like plant height, leaf length, leaf wide, leaf area, leaf number, leaf area index, stem diameter, number of root and growth parameters viz. crop growth rate, relative growth rate, chlorophyll content in leaf were affected by spray formulation. The effect of cultivars on those morpho-physiological and growth features also significant. Data revealed that increasing plant height, leaf wide, leaf area, leaf number, leaf area index, stem diameter, number of root and RGR was higher in Mokara Yellow Anne. CGR, leaf length, total chlorophyll content were higher in Mokara Diheard red.

Different Spray formulation with different ratio of $\mathrm{N}, \mathrm{P}$ and $\mathrm{K}$ also significantly affected morphophysiological features and growth parameters. Leaf length was slightly affected by spray formulation. Degrees of increasing leaf length, number of root were higher in spray formulation-1 $\left(\mathrm{F}_{1}\right)$. Rate of increasing of plant height, crop growth rate, relative growth rate were more in spray formulation-2 $\left(\mathrm{F}_{2}\right)$. Rate of increasing of leaf width, total leaf area, leaf number, leaf area index, stem diameter and total chlorophyll content were more in spray formulation-3 $\left(\mathrm{F}_{3}\right)$.

Plant height, stem diameter, total leaf area and CGR were best in $\mathrm{V}_{2} \mathrm{~F}_{2}$ among all treatments. Leaf area, leaf area index and RGR were best in $\mathrm{V}_{2} \mathrm{~F}_{3}$ among all treatments. Leaf length and leaf width were best in $\mathrm{V}_{2} \mathrm{~F}_{1}$ and $\mathrm{V}_{2} \mathrm{~F}_{3}$, respectively. Chlorophyll content was superior in $\mathrm{V}_{1} \mathrm{~F}_{3}$ among all treatments.

Based on experimental results it may be concluded that

i) The morphological and physiological attributes was significantly affected by spray formulation.

ii) Spray formulation-2 $\left(\mathrm{F}_{2}\right)$ was the best for growth of orchid.

iii) The ratio of N, P and K at 10:15: 20, 15:20:25, 10:25:25, and 10:25:30 for spray formulation might be the best for growth of orchid.

\section{Acknowledgement}

The author is grateful to the Omnipotent and Almighty God, the supreme ruler of the universe, for his immense blessing to keep us alive and successful completion of this research work.

The author likes to express profound gratitude, deepest respect and most sincere appreciation to his supervisor Prof. Dr. M. Obaidul Islam, Department of Crop Botany, Bangladesh Agricultural University (BAU), Mymensingh for his scholastic guidance, supervision, constant encouragement and suggestion and intellectual comments throughout the progress of this research work and preparing the manuscript. 
The author expresses deep sense of gratitude and most sincere appreciation to his honorable cosupervisor Prof. Dr. A.K.M. Azad-ud-doula Prodhan Department of Crop Botany, Bangladesh Agricultural University (BAU), Mymensingh for his, suggestion, encouragement, constructive criticism and inspiration during the period of research work

The author expresses special thanks to Md. Iquebal Kabir, Ripon Kumar Gharmi, S. M. Kamrul Hasan, Harun-ar-Rashid, Md.Yousuf Ali, for their kind help during the period of the research work.

Thankfully acknowledgement is due to United State Department of Agriculture (USDA) for giving partly financial support in present research under the project "In vitro regeneration of orchids for commercial production and conservation of endangered species" (Project No. 2008/79/USDA).

Finally, the author expresses his sincere gratitude to beloved parents, brothers and sisters whose sacrifice and encouragement have been paved the way during study period

\section{References}

[1] Chandra, S. and Das, A. K. 2000. Correlation and interrelation of physiological parameters in rice under rainfed transplanted condition. J. Crop Res. Assam Agric.Univ., 19(2): 252 - 254

[2] Chowdhury, M. 1975. "Baldah Garden", Dhaka, Bangladesh. pp. 12-15.

[3] Goh, C. J., Sim, A. A. and Lim. G. 1992. Mycorrhizal associations in some tropical orchids. Lindleyana, 7(1):13-17. Hatch, E. D. 1953. Orquideas Subterranean., 15 (1): 2-11.

[4] Gomez, A. K. and Gomez, A. A. 1984. Statistical Procedures for Agricultural Research, 2nd Ed., John Wiley and Sons, Inc., NY. pp. 8-20.

[5] Hagakl, Y. and Imamura, J. S. 1987. NPK requirements of vanda miss Joaquin orchid plants. Department of Horticulture. Hawaii Institute of Tropical Agriculture and Human Resources. University of Hawaii. Hilo.pp. 34 - 36.

[6] Kuksal, R. P, Singh, R.D and Yadar, N. 1997. Effect of different level of nitrogen and phosphorous on fruit and seed yield of tomato.Prog. Hort., 9(2): 13-20.

[7] Patil, P.V. 2001. Orchids of India in Commercial Flowers $2^{\text {nd }}$ Ed. Calcutta, India.Pp 193.

[8] Russel, D. F. 1986. MSTAT-C.MSTAT Director. Crop and Soil Science. Department of Michigan State University, USA.

[9] Singh, R. and Kohli, A. 1999. Effect of N, P and K regimes on growth and development of orchid .J. Hill Res., U.K. 12(1): 63 66.

[10] Singh, B. and Voleti, S. R. 1995.Research and achievements. Division of Floriculture and Landscaping. Indian Agricultural Research Institute, New Delhi. p.15.

[11] Sharma, S. K. and Haloi, B. 2001. Characterization of crop growth variable in some selected rice cultivars of Assam. Indian J. Plant Physiol., 6: $166-171$. 